\title{
Seismic damage and destructive potential of seismic events
}

\author{
Donato Abruzzese $\left({ }^{1}\right)$ and Stefano Maria Petrazzuoli $\left({ }^{2}\right)$ \\ $\left({ }^{1}\right)$ Dipartimento di Ingegneria Civile, Università «Tor Vergata», Roma, Italy \\ $\left.{ }^{2}\right)$ Osservatorio Vesuviano, Napoli, Italy
}

\begin{abstract}
This paper has been written within a research framework investigating the destructive potential of seismic events. The elastic response spectra seem insufficient to explain the behaviour of structures subject to large earthquakes in which they experience extensive plastic deformations. Recent works emphasise that there were many difficulties in the definition of a single parameter linked to the destructive potential of an earthquake. In this work a study on the effect of frequency content on structural damage has been carried out. The behaviour sults obtained suggest a method for evaluating analysed, considering several artificial earthquakes. The reresponse spectra ad the frequency content of the destructive seismic potential of an earthquake through the structures within the analysed area.
\end{abstract}

Key words seismic destructive potential - frequency content - elasto-plastic oscillator - response spectra

\section{Introduction}

Damage charts have been widely utilised in historical seismicity to illustrate the actions and intensities of the seismic event, evaluated «a posteriori» and related to the damage to the built-up environment.

It is understandable that the information obtained from these maps correlates the seismic action with the observed effect on a certain class of buildings, and is not directly valid for a generic intensity evaluation of ground-shaking. In fact, the damage suffered by constructions differs, being a function not only of the material resistance of the elements in the structure, but also of their dynamic properties (Abruzzese and Como, 1993), and in most cases this means their vibration period (Abruzzese and Petrazzuoli, 1993). Since it is possible to record a seismic event and to analyse it, producing response spectra of the signal, in terms of acceleration, velocity ad displacement, researchers are able to evaluate the seismic action intensity as a function of frequency content. Frequency content is most important for the evaluation of expected damage of different building classes, grouped by their dynamic behaviour, as are the vibration period, the resistance level and allowable ductility.

All this is of great importance, and the most recent results show a correlation between the seismic destructive potential, representative of the global effect of the event, the duration of the event, its frequency content, and the peak level of the ground acceleration.

The damage parameters proposed by some researchers are based on seismic parameters that seem to be correlated with the damage suffered by the building, and do not take into account the real behaviour of the structure; particularly the structural decay connected with a seismic action, as recently shown by Bertero (1989).

De Pasquale and Cakmak (1989) suggest a method to evaluate the integrity of a structure after a strong earthquake. They compare the values of the vibration period of the structure 
before and after the damage, assuming the «softening» $d$ as the main damage parameter:

$$
d=1-T_{\mathrm{init}} / T_{\max } .
$$

Chang and Franklin (1987) analyzed the correlation between reported damage (MMI, Mercalli Modified Intensity Scale) and the PGA, RMSA and PSDF (Power Spectrum Density Function) parameters, with particular reference to the local response. Alter the manipulation of 421 seismic records, most of them recorded in Italy, they point out that only the PSDF parameter seems, in some way, to agree with the extensive damage which occurred to the buildings on soft soil. Chiostrini et al. (1991) pointed out that even masonry buildings can change their vibration period remarkably during a seismic action, using the results obtained in a dynamic test of a full scale masonry structure.

The dynamic analysis of the structure, performed via linear-elastic analysis (elastic response spectra), seems insufficient to give reliable results concerning the dynamic behaviour in the case of earthquakes of medium-strong intensity, particularly when the deformations are large, causing parts of a structure to reach a plastic state.

\section{Modelling the elasto-plastic oscillator}

Generally speaking, it is usually possible to model a real structure as an elasto-plastic oscil-

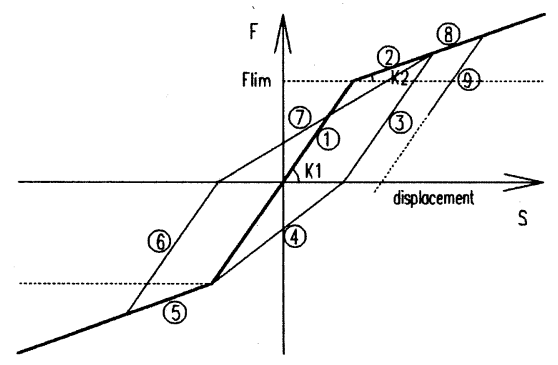

"A" MODEL (TAKEDA) lator, assuming that the elastic state of the structure is the state in which plastic hinges have not yet appeared, while the plastic state is that in the which the structure contains a large number of elements in the plastic state. Different types of elasto-plastic oscillators can be adopted to model non-linear behaviour, or, in other words, the behaviour of structures beyond the elastic limit, during a dynamic action.

The models with decay, which match the structure collapse with maximum plastic deformation, are most used for reinforced concrete structures (Takeda, 1988), and are usually the most reliable. Such models depend on loading and unloading cycles and include a stiffness reduction during the loading process (A model) (fig. 1).

We can observe that the characteristic path of the oscillator follows a bi-latera, in which the stiffness is equal to the elastic stiffness $(\mathrm{Ks}=\mathrm{K} 1)$ for every load cycle (1)(5), while the unloading cycle (2)(7) follows a path with a stiffness $\mathrm{Kc1}$ and $\mathrm{Kc} 2$ which are monoton decreasing functions of the limit values $x_{\text {lim }}$, $F_{\text {lim. }}$. After each step over the elastic limit (plastic state) the limit values $x_{\lim }$, and $F_{\text {lim }}$ are modified.

A second model with decay, which is less common, is the so called «origin-oriented» (B model). Such a model seems more effective to model reinforced concrete buildings or masonry buildings with decay due to shear mech- 
anisms (Cosenza and Manfredi, 1991). The plastic side of the model is almost horizontal, and every step over the elastic limit modifies the limit value for the deformation $x_{\lim }$ (softening).

The load and unload paths are characterised by the same stiffness and by a lack of plastic residual deformation (passing through the origin). It seems evident that such a model allows a very low energy quantity to be dissipated.

\section{The frequency content effect on the structural damage}

The first approach to the problem has been a study of the dynamic behaviour of an elastoplastic oscillator type $\mathrm{A}$ and $\mathrm{B}$, and the effects of the frequency content on the structural damage. In this first case the shape of the external actions has been assumed to be a perfect «sin» function, having the intensity of:

$$
F_{e}(t)=F_{\lim } \cdot \sin \left(\frac{2 \pi}{T} \cdot t\right)
$$

where $F_{\text {lim }}$ means the elastic limit force for the oscillator. The structural damping has been assumed equal to $5 \%$. The parameter characterising the decay of the oscillator is the well known kinematic ductility:

$$
\mu=x_{\max } / x_{\lim }
$$

where $x_{\lim }$ is the limit elastic displacement and $x_{\max }$ the maximum displacement.

The limited validation of this parameter as a damage functional is well known (Cosenza et al., 1991), but we considered it more convenient to use this function in order to define in a simple way the damage to a structure.

Figure 2a-d shows the response curves of several elasto-plastic oscillators having initial oscillating period $\left(T_{\text {init }}\right)$ in the range 0.3-0.7 $\mathrm{s}$ for model $\mathrm{A}$ and $0.1-0.5$ for model $\mathrm{B}$. The results also show values of the kinematic ductility.

This figure illustrate that the periods inducing a greater damage level $\left(T_{\text {peak }}\right)$ may be very different from the initial vibration period value
( $\left.T_{\text {init }}\right)$. The values of the $T_{\text {peak }} / T_{\text {init }}$ rate obtained by starting from the $\mathrm{K} 2 / \mathrm{K} 1$ rate, from the vibration period of the oscillator and depending on the model types $\mathrm{A}$ and $\mathrm{B}$, are shown in table I.

The decay effect, as a stiffness reduction, makes a frequency content of approximately $1 \mathrm{~s}$, and a slightly greater low frequency induces considerable damage even on framed reinforced concrete buildings, of medium-low height (4-8 floors) with high stiffness, or on masonry buildings having a vibration period shorter than $0.5 \mathrm{~s}$.

To produce a strong decay action on an oscillator it is necessary for the seismic action to have a high intensity with a period of around 1.5-2 times the value $T_{\text {init }}$. In order to verify the results and the remarks made for the analysis of a perfect sinusoidal external action an analysis has been performed which considers a real seismic action. In this case, a signal of 5 different accelerograms was artificially generated and the non linear response spectra of an oscillator was evaluated as previously. The five seismic actions considered have a different frequency content.

The first and second actions correspond to the spectra $\mathrm{S} 1$ and $\mathrm{S} 2$, as suggested by the National Italian Group for the Defense against the Earthquakes (GNDT, 1984); the third is characterised by periods ranging from 0.8 and $1 \mathrm{~s}$, the fourth with periods ranging from 0.5 and $1 \mathrm{~s}$, and the fifth with periods ranging from 0.5 and $1.5 \mathrm{~s}$ (fig. 3). It is interesting to note that the spectrum S1, characterised by an action stronger in the lower periods, represents the expected effect on a structure lying on a rock, while the spectrum $\mathrm{S} 2$ represents the analogous effect expected for a structure lying on a softer soil.

We use artificial accelerograms, having the same duration and amplitude function, instead of real ones to emphasise the effect of the frequency content on the structural damage.

Figure 4 shows the response spectra of elastoplastic oscillators with behaviour model $\mathrm{A}$ and model $\mathrm{B}$, corresponding to the 5 artificial seismograms considered. Model A represents the behaviour of reinforced concrete structures, with destructive periods in the range 

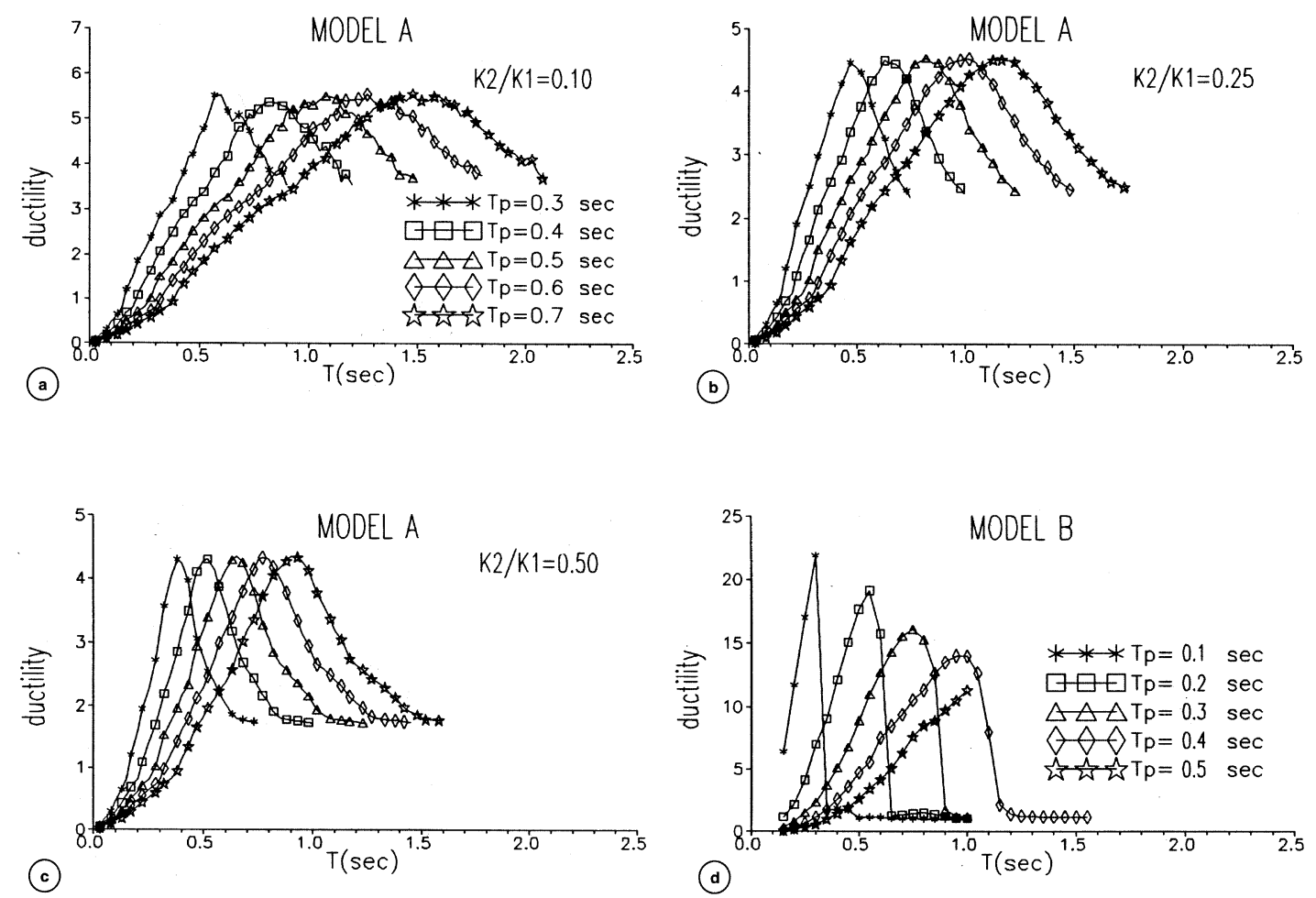

Fig. 2a-d. Response curves for elasto-plastic oscillators with natural initial period $\left(T_{\text {init }}\right)$ variable in the range of 0.3 and $0.7 \mathrm{~s}$ for the reinforced concrete structure $(\mathrm{a}, \mathrm{b}, \mathrm{c})$ and in the range of 0.1 and 0.5 for masonry building (d).

Table I. Value of the $T_{\text {peak }} / T_{\text {init }}$ rate versus the $\mathrm{K} 2 / \mathrm{K} 1$ rate and initial vibration period (for model A) and versus the initial vibration period (for model B).

\begin{tabular}{|c|c|c|c|c|c|}
\hline \multicolumn{6}{|c|}{ Rate $T_{\text {peak }} / T_{\text {init }}$} \\
\hline \multirow{2}{*}{$\frac{\mathrm{K} 2 / \mathrm{K} 1}{T_{\text {init }}}$} & \multicolumn{3}{|c|}{ Model A } & \multicolumn{2}{|c|}{ Model B } \\
\hline & 0.1 & 0.25 & 0.5 & $T_{\text {init }}$ & \\
\hline 0.3 & 2.33 & 1.57 & 1.27 & 0.1 & 3.00 \\
\hline 0.4 & 2.20 & 1.58 & 1.30 & 0.2 & 2.75 \\
\hline 0.5 & 2.26 & 1.64 & 1.30 & 0.3 & 2.50 \\
\hline 0.6 & 2.25 & 1.67 & 1.28 & 0.4 & 2.37 \\
\hline 0.7 & 2.33 & 1.64 & 1.33 & 0.5 & 2.20 \\
\hline
\end{tabular}

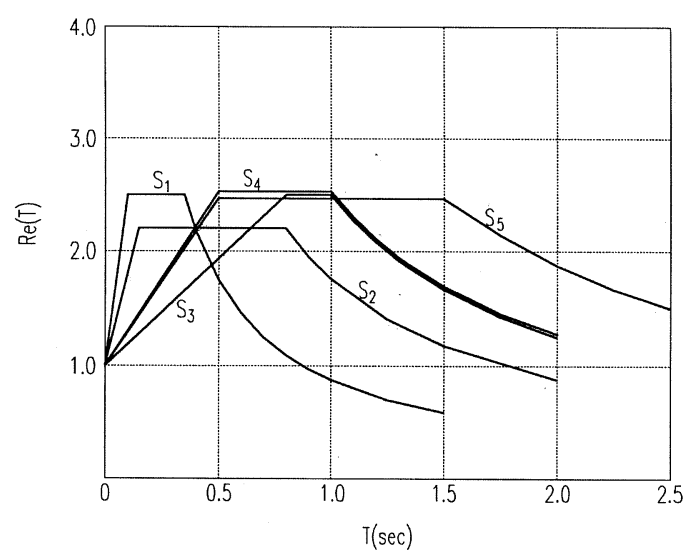

Fig. 3. Spectral shape of the artificially generated earthquakes. 


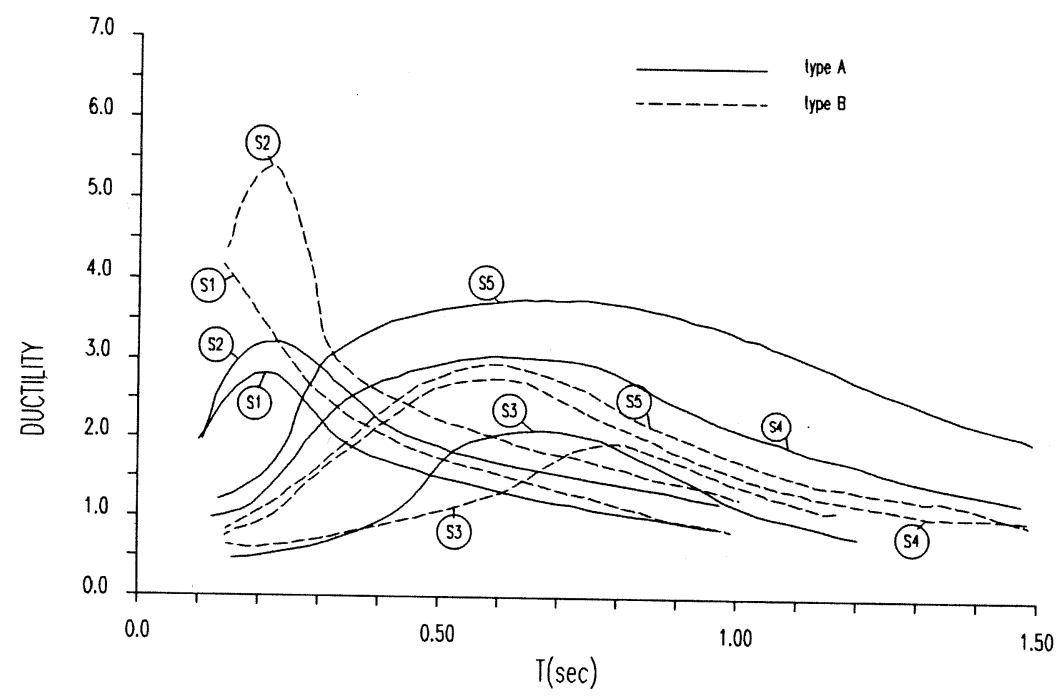
Fig. 4. Comparison between the response spectra of the oscillators A and B, in term of the kinematic ductility
request by the $i$-th seismic action.

0.5-1.5 s. The events with a greater ductility demand are S5 and S4, while the effects of the $\mathrm{S} 1$ and $\mathrm{S} 2$ earthquakes are relatively low.

For model B, representing the behaviour of a considerable number of masonry structures, the most destructive actions are S1 and S2. It is possible to state that for this model, and hence for masonry buildings, the range of the destructive periods corresponds to the interval $0-0.5 \mathrm{~s}$.

However, the curves corresponding to models A and B are not strictly comparable, because for type B oscillators the seismic action has been reduced by $30 \%$.

Indeed, when applying the whole value for the seismic action considered, the ductility demand is too high to plot both response curves for the two models on the same diagram.

A lower ductility demand occurs for both models $\mathrm{A}$ and $\mathrm{B}$ during the $\mathrm{S} 3$ seismic action.

\section{Conclusions}

This paper shows the importance of the frequency content on the destructive power which is related to a seismic action.
The correct design for a building exposed to strong seismic actions must take into account two fundamental aspects of the whole phenomenon.

The first is the non linear behaviour of the structure, related to the elasto-plastic behaviour and to the obvious consequent decay; the second is the frequency content of the seismic action at ground level.

In the analysis described here, we have shown that in the case of reinforced concrete buildings, which have a vibration period in a range between 0.3 and $0.7 \mathrm{~s}$ the periods between 0.5 and $1.5 \mathrm{~s}$ are the most destructive.

However if the vibration period is smaller, that is to say smaller than $0.3 \mathrm{~s}$ (this is the case with masonry buildings (Foraboschi et al., 1993) or less stiff r.c. buildings), the critical interval is $0-0.5 \mathrm{~s}$.

This suggests that one method to evaluate the destructive power $\left(P_{d}\right)$ of seismic events might be to integrate the acceleration spectra in a critical interval $T_{1}-T_{2}$ of the period,

$$
P_{d}=\int_{T_{1}}^{T_{2}} S_{a}(T) \cdot d T
$$


According to the presented analysis we can state that the value of the periods $T_{1}$ and $T_{2}$ approximately are:

- for masonry buildings:

$$
T_{1}=0 ; \quad T_{2}=0.5 \mathrm{~s}
$$

- for reinforced concrete buildings:

$$
T_{1}=0.5 ; \quad T_{2}=1.5 \mathrm{~s} .
$$

Furthermore it can be shown that strong seismic actions, characterised by a narrow band frequency content, as for the S3 action, may produce a reduction in damage, while a moderate intensity seismic action, with a large band frequency, may produce greater damage.

We accept that the real behaviour of buildings is more complex because some important parameters not well known, for example, the allowable actual ductility value and the presence of the phenomena of fragility, which applies mainly to masonry buildings.

We also recognise that the elasto-plastic oscillator models utilised in the paper are fairly simple, but the aim of the research was to show the effect of the frequency content on potential structural damage.

\section{REFERENCES}

AbruzZese, D. and M. Como (1983): Sulla vulnerabilità sismica delle costruzioni ed effetti della scuotibilità dei siti, in $X V$ Convegno Nazionale di Geotecnica, Spoleto, 79-84.
Abruzzese, D. and S. Petrazzuoli (1993): Valutazione dell'effetto del contenuto in frequenza di un'azione sismica sul danno strutturale di un oscillatore elastoplastico, in $6^{\circ}$ Convegno ANIDIS L'Ingegneria Sismica in Italia, Perugia, 55-62.

BERTERO, V. V. (1989): Lessons learned from recent catastrophic earthquakes and associated research, in Primera Conferencia International Torroja 1989. Madrid.

Chang, F.K. and A.G. Franklin (1987): «PGA, RMSA, PSDF, Duration and MMI», Ground Motion and Engineering Seismology (Elsevier, Amsterdam), 449-465.

Chiostrini, S., P. Foraboschi, A. Vignoli, C. GalimBerti, M. Meneghella and P. Penzeri (1991): Prove di vibrazioni forzate su di un edificio in muratura. Danneggiamento e non linearità della risposta, in $5^{\circ}$ Convegno L'Ingegneria Sismica in Italia, Palermo, 601-610.

Cosenza, E. and G. MANFredi (1991): Un modello evolutivo-degradante per l'analisi del danneggiamento strutturale sotto azioni sismiche, in $5^{\circ}$ Convegno L'Ingegneria Sismica in Italia, Palermo, 93-102.

Cosenza, E., G. MAnfredi and R. Ramasco (1991): L'influenza della modellazione strutturale nella valutazione del danneggiamento per azioni sismiche, in $5^{\circ}$ Convegno L'Ingegneria Sismica in Italia, Palermo, 103-116.

De Pasquale, E. and A. Cakmak (1989): Parameterbased damage indices and detection of seismic structural damages, in Proceedings 4th International Conference on Computational Methods and Experimental Measurements, Capri.

Foraboschi, P., S. Sorace and A. Vignoli (1993): Modellazione dinamica di edifici esistenti in muratura, in $6^{\circ}$ Convegno ANIDIS L'Ingegneria Sismica in Italia, Perugia, 401-408.

GNDT (1984): Norme tecniche per le costruzioni in zona sismica, in Convegno Nazionale Ingegneria Sismica, Rapallo.

TAKEDA, J. and H.H. Komoto (1988): Deteriorative rate effects on brittle fractures of reinforced concrete structures during severe earthquakes, in Proceedings 9th World Conference on Earthquake Engineering, Tokyo. 